\title{
Character Theory of Finite Groups
}

\author{
January-March 2009 \\ EXERCISE SHEET 3
}

Starred questions are somewhat harder. Do not attempt questions 6 and 7 until character triples and the Schur multiplier have been introduced in lectures.

1. (i) Suppose $\mathcal{K}$ is a conjugacy class of $S_{n}$ contained in $A_{n}$; then $\mathcal{K}$ is called split if $\mathcal{K}$ is a union of two conjugacy classes of $A_{n}$. Show that the number of split conjugacy classes contained in $A_{n}$ is equal to the number of characters $\chi \in \operatorname{Irr}\left(S_{n}\right)$ such that $\chi_{A_{n}}$ is not irreducible. (Hint. Consider the vector space of class functions on $A_{n}$ which are invariant under conjugation by the transposition (12).)

(ii) Let $g \in A_{n}$ have a cyclic decomposition with cycle lengths

$$
\mu_{1} \geq \mu_{2} \geq \cdots \geq \mu_{k}>0
$$

Show that the conjugacy class of $g$ in $S_{n}$ is split if and only if the numbers $\mu_{i}$ are all distinct and odd. Deduce that the number of partitions $\lambda$ of $n$ such that $\lambda=\lambda^{\prime}$ is equal to the number of partitions $\left(\mu_{1}, \ldots, \mu_{k}\right)$ of $n$ with all parts $\mu_{i}$ distinct and odd.

(iii)* Find an explicit combinatorial one-to-one correspondence between the set of self-conjugate partitions of $n$ and the set of partitions of $n$ with all parts distinct and odd.

2. Let $\chi$ be a character of $G$ and let $p$ be a prime. For $g \in G$, write $g=g_{p} g_{p^{\prime}}=g_{p^{\prime}} g_{p}$ where $g_{p}$ is a $p$-element and $g_{p^{\prime}}$ is $p$-regular. Define $\theta(g)=\chi\left(g_{p^{\prime}}\right)$. Show that $\theta$ is a generalised character of $G$.

3. Let $|G|=p^{k} m$ where the prime $p$ does not divide $m$. Suppose $\chi \in \operatorname{Irr}(G)$ and $\chi(1)$ is divisible by $p^{k}$. Define a class function $\theta: G \rightarrow \mathbb{C}$ by

$$
\theta(g)= \begin{cases}\chi(g) & \text { if } g \text { is } p \text {-regular } \\ 0 & \text { otherwise }\end{cases}
$$

(i) Let $g \in G$ and write $\left|C_{G}(g)\right|=p^{l} s$ where $p$ does not divide $s$. Prove that $p^{-l} \chi(g)$ is an algebraic integer. (Hint. Consider the map $\omega_{\chi}$. Also, use the following easy fact: if $a \in \mathbb{C}$ and both $a u$ and $a v$ are algebraic integers for some coprime $u, v \in \mathbb{Z}$ then $a$ is an algebraic integer.)

(ii) Let $E$ be a $q$-elementary subgroup of $G$ where $q$ is any prime. Write $E=P Q$ where $P$ is a $p$-group, $Q$ is a $p^{\prime}$-group and $P \cap Q=[P, Q]=\{1\}$. Prove that $|P|^{-1} \chi_{Q}$ is a character of $Q$. Hence show that $\theta_{E}$ is a character of $E$.

(iii) Deduce that $\theta$ is a generalised character of $G$.

(iv) Show that $\chi(g)=0$ for all $g \in G$ satisfying $p \mid o(g)$. (Hint. Consider $\langle\theta, \chi\rangle$.) 
4. (Mackey formula) Let $H$ and $K$ be subgroups of $G$. Let $T$ be a set of representatives of double $H-K$ cosets; that is,

$$
G=\bigcup_{t \in T} H t K
$$

is a disjoint union. Let $\theta$ be a class function on $H$. Show that

$$
\left(\theta^{G}\right)_{K}=\sum_{t \in T}\left(\theta_{H^{t} \cap K}^{t}\right)^{K}
$$

where as usual $\theta^{t}$ is the class function on $H^{t}$ given by $\theta^{t}(x)=\theta\left(t x t^{-1}\right), x \in H^{t}$.

5.* Consider the group $\mathrm{GL}_{2}(q)$ of all invertible $2 \times 2$ matrices with entries in $\mathbb{F}_{q}$. Let $\alpha$ and $\beta$ be distinct linear characters of the multiplicative group $\mathbb{F}_{q}^{\times}$. Let $B$ be the subgroup of $\mathrm{GL}_{2}(q)$ consisting of all invertible upper-triangular matrices. Define the linear character $\phi=\phi_{\alpha, \beta}$ of $B$ by

$$
\phi\left(\left(\begin{array}{ll}
x & z \\
0 & y
\end{array}\right)\right)=\alpha(x) \beta(y)
$$

Prove that $\phi^{G}$ is irreducible. Show that $\phi_{\alpha, \beta}^{G}=\phi_{\alpha^{\prime}, \beta^{\prime}}^{G}$ if and only if either $\alpha=\alpha^{\prime}$ and $\beta=\beta^{\prime}$ or $\alpha=\beta^{\prime}$ and $\beta=\alpha^{\prime}$.

Hint. Use the Mackey formula.

6. Suppose $\left(\operatorname{Id}_{G}, \sigma\right)$ is a strong isomorphism of the character triple $\left(G,\{1\}, 1_{\{1\}}\right)$ onto itself, where $\operatorname{Id}_{G}: G \rightarrow G$ is the identity map. Show that there exists a linear character $\lambda$ of $G$ such that, for all subgroups $H \leq G$ and all characters $\chi$ of $H, \sigma_{H}(\chi)=\chi \lambda_{H}$.

7. Let $C$ be a cyclic group. Prove that the Schur multiplier $M(C)$ is trivial.

ae284@cam.ac.uk 